\title{
Research on technical support SaaS platform of soil pollution control based on the whole process management
}

\author{
Yuanhua Chen*, Hao Wang, and Tianwei Sun \\ CEPEC HRI Technology Co., Ltd, Beijing, 10082, China
}

\begin{abstract}
In order to improve the effect of soil pollution prevention and control, a technical-support cloud platform is designed to establish a high-level technologies, uniform standards, and regulatory tool for soil environmental investigation, risk assessment and repair work. Firstly, according to the process of soil remediation, combined with the source and pollution characteristics of soil pollutants, the main pollution components in the soil environment were classified and distributed, and then the platform was designed and developed. The system has the functions of project management, query, risk analysis and prediction, drawing, tabulation and so on. Finally, combining with the soil environmental quality standards, the effectiveness and cost indicators of the treatment methods were classified and digitized, which would greatly improve the implementation efficiency and reduce the cost of the project management.
\end{abstract}

\section{Introduction}

In consideration of the soil providing basic material guarantee for human survival and activities, the soil environmental quality is a people's livelihood issue. Once the land is polluted, it must bring great threat to the quality of agricultural products and human health, concerning the survival security of future generations [1]. And it is gradually realized that the soil pollution prevention and treatment is a key segment to construct a modern environmental governance system and governance capacity [2], an important carrier to grasp that lucid waters and lush mountains are invaluable assets, and a significant practice to implement $\mathrm{Xi}$ Jinping's socialist ideology with China's characteristics in the new era.

Soil pollution in China is a long-term accumulation with the economic and social development process. However, the prevention and control of soil pollution started relatively late, the development of the whole industry system is not yet mature. For achieving the great-leap-forward development, it is necessary to use the idea of system engineering to carry out top-level design and planning from the global and strategic height. In recent years, China has successively introduced and implemented a series of laws and regulations, such as Action Plan for Soil Pollution Prevention and Control, Law of the People's Republic of China on Soil Pollution Control, Environmental Protection Law of the People's Republic of China on Solid Waste Pollution, the task of investigating soil environmental quality and establishing soil environmental governance system is becoming urgent.

Currently, the soil pollution prevention and control work in China is still in preliminary development phase, there are still some problems urgent to be solved. First of all, soil remediation has a certain degree of complexity and systematic [3]. The characteristics of the projects organization and implementation in China, as extensive region, great difficulty, long cycle, tight disposal resources and time, numerous regulations, and high environmental protection requirements, lead much work carried out in the case of unclear, inaccurate and incomplete information, causing supervision blind, inverted links and fuzzy results [4]. The serious consequence caused by this situation may extend the investigation and evaluation cycle, weaken the intensity of engineering supervision, reduce the scope of quality control, increase the cost of restoration work, and even bring more serious secondary pollution. Furthermore, because existing soil protection management method backwardness, monitoring data, investigation and assessment reports, construction records have to be retained in paper version, some questions in data integrity, accuracy and reliability have been exposed, hindering the work of implementation process management, retroactive liability, and post-implementation evaluation. And then, the small proportion of practitioners and institutions in soil monitoring, environmental surveys, risk assessment and remediation, as well as the irregularity technical level, cannot meet the existing needs, the contamination control efficiency is very urgent to enhance [5].

Overview of China's soil pollution prevention and control work, the development path is gradually from the initial end pollution control to the current soil restoration based on residents, health and social harmony, and finally towards the co-ordinate system

\footnotetext{
*Corresponding author: chenyuanhua@cepec.cn
} 
building a harmonious coexistence of human and urban natural ecology.

With the deep integration of Internet technology and industry, we proposes a technical support Software as a Service (SaaS) platform for the whole process of soil pollution prevention and control, constructing an intelligent technical system to promote the whole life cycle coordination and guidance of soil pollution investigation and judgment, risk identification and evaluation, governance and remediation, so as to improve the efficiency and effectiveness of soil pollution control integrally. The use of the platform will help the soil remediation designer, engineer and manager obtain comprehensive data to complete their task, and also all process information will be recorded in the system.

\section{Design plan}

\subsection{Objective}

According to the design and implementation process of the project, main objectives of the platform are as follows:

- Establish a database for standardized data storage in the process of project management.

- Establish a set of physical and chemical parameters of common pollutants in characteristic pollutants database. - Establish a pollution remediation technology library, classified by technical principle, characteristics and application scope, to support the screening of engineering design process.

- Design laboratory data acquisition and analysis tools to support project data management and data analysis.

- Design data management tools for the establishment of pollution site archives.

- Design convenient preliminary investigation tools to assist preliminary investigation and accelerate the pollution identification progress.

- Design unified, standard pollution analysis and risk assessment methods or models.

- Provide data and technical support for the management of contaminated site remediation projects.

- Using WebGIS technology to visualize the status of contaminated sites and related information.

\subsection{Framework design}

The technology platform is composed of perception layer, cloud platform, technical support layer (AI and big data), and application layer, forming an integrated management and control architecture (as shown in Figure 1).

The perception layer is the sensory organ, focusing on the environmental information acquisition and identification.

With the applying cloud-storage platform to the construction of project storage system, the efficient storage and retrieval of project information are achieved.

Technical support layer through the data engine, GIS engine, service components support module as a bridge and link between the applications and the data layer.

The application layer provides requesting / responsing clients, as well as dynamically generates web page and so on. Clients use the apps to support their design and analysis .

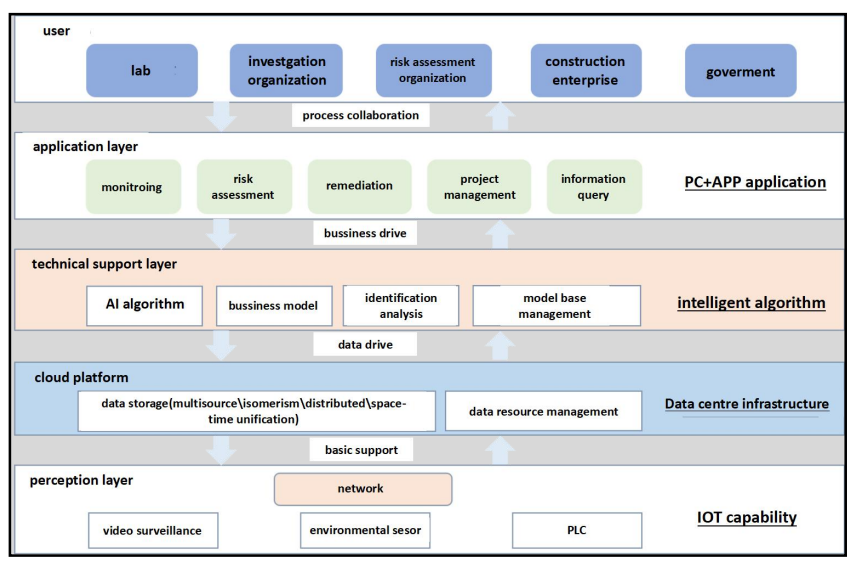

Fig. 1. Platform framework.

\subsection{Function design}

In view of contaminated site remediation boundary and process, the platform was designed for various types of soil pollution projects' survey, risk assessment and remediation, as construction land, agriculture land,mining land and river land. The main functions of the platform are as follows.

\subsubsection{Project management}

Based on the function, the management system are divided into management module of basic information function, real-time monitoring of processing module, function module to apply for approval, the statistics query management module.

\subsubsection{Preliminary investigation on contaminated site}

Based on data obtained from field soil investigations and laboratory analysis, the present situation and temporal and spatial changes of soil pollution can be preliminarily judged.

\subsubsection{Detailed Investigation on contaminated site}

The module integrates digital terrain analysis, geographic information system (GIS) and soil survey techniques for soil pollution prediction.

\subsubsection{Environmental risk assessment}

By applying probability statistics, a comprehensive evaluation has been made on the pollution risk for different production, pollution sources and environmental function areas. 


\subsubsection{Risk management and control}

The application of risk management in the system is to deal with the accidental pollution risk, such as avoiding and preventing known risk, mitigating and changing the source of risk and building up emergency plan.

\subsubsection{Decision support}

Based on historic cases, the module can provide some useful and referenced results, as repairing goal, repairing approach and management method.

As shown in Figure 2, the working procedure of the platform is started from project management, then followed by site preliminary investigation, site detailed investigation, environmental risk assessment and environmental risk control. It can process multiple projects at the same time, and one projects can be recorded with only one link information.

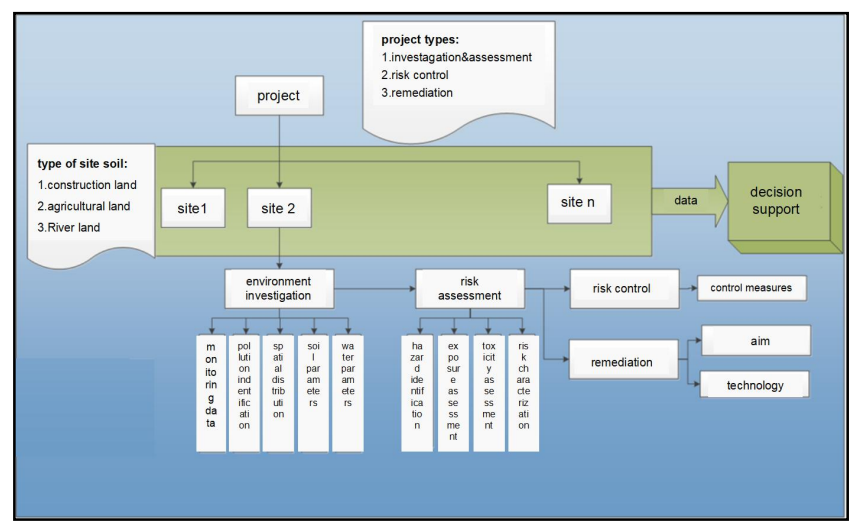

Fig. 2. System function.

\section{Implementation}

According to the function design, the platform is developed as follows.

When the soil properties and the degree of contamination was studied with statistic method, samples were entirely independent and obeyed normal distribution, taking into account spatial relative of sampling location (as shown in Figure 3). The sampling information contains the sampling point location, altitude, land use types, soil types, physical and chemical elements, inorganic elements and so on.

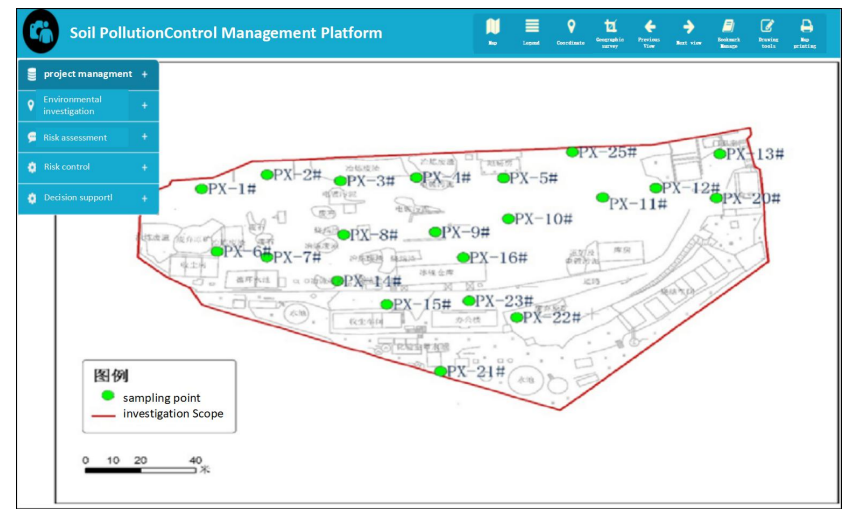

Fig. 3. Sampling location.
Taking Huanjiang County as the survey area, the feasibility of soil pollution management is analyzed based on system study of soil pollution sample point using geostatistics method supported by GIS technology, to carry out site environmental survey and environment risk assessment. Users could view the outcome of assessment directly by viewing a grid map in form of GIS, which is colored with various hues according to the evaluation rankings (as shown in Figure 4).

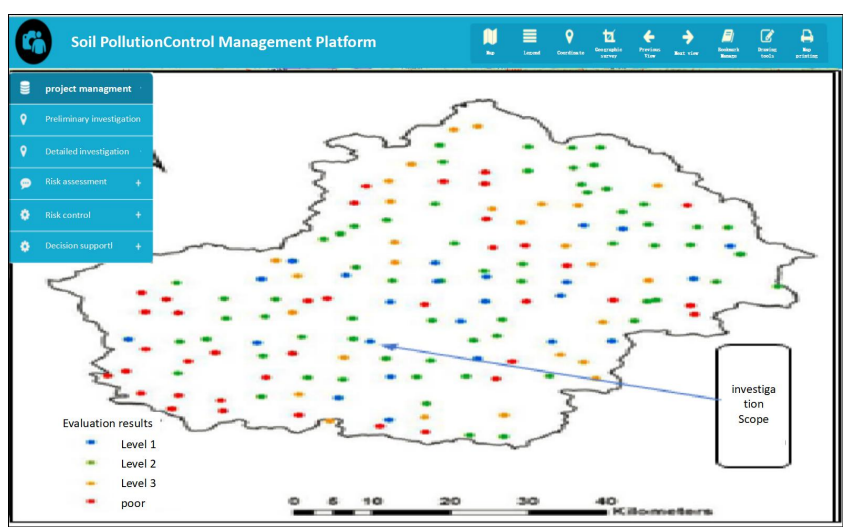

Fig. 4. Risk calculation result.

Interpolation calculation is the most important calculating job of a SaaS platform, which is the key technology to select one proper interpolation method to ensure the real-time and accurate character of the system. Based on soil pollution sampling data, soil pollution sampling number was established based on different confidence levels and different precision coefficient in Huanjiang County, and interpolation calculation was conducted based on several interpolation methods (as shown in Figure 5).

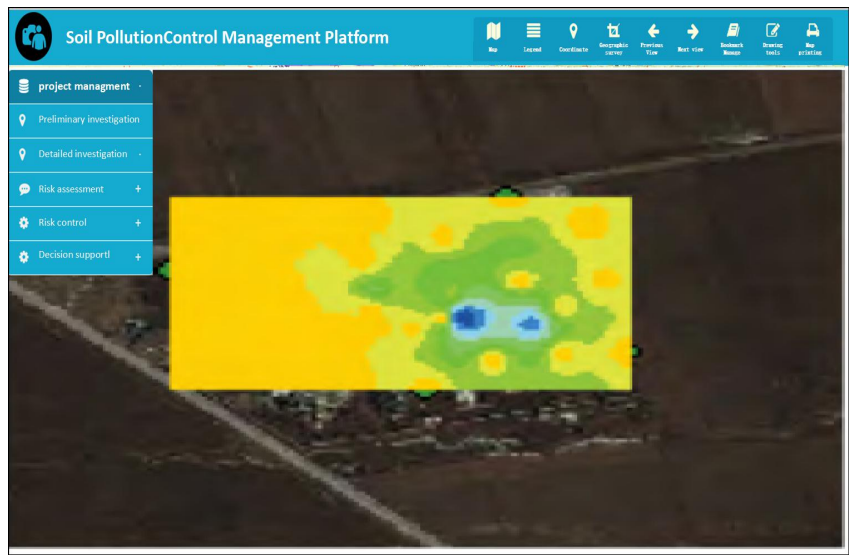

Fig. 5. Interpolation calculation results.

\section{Conclusion}

Referring to the requirements of national soil pollution control and the demand of the project management, the platform comprehensively uses advanced technologies such as GIS, big data and mobile Internet to record the construction of soil pollution control. And the establishment of soil pollution prevention and control technology support system provides advanced risk control means for the 
construction of soil pollution prevention and control pilot area, which has strong demonstration significance.

\section{References}

[1] Scientific planning of '12th Five-Year' soil environmental protection work [J]. Environmental Protection. 2011 (13).

[2] ZHAI Qing. Promoting the Modernization of Ecological Environment Management System and Management Ability to Provide Strong Guarantees for the Battle of Pollution Control [J]. China Institutional Reform and Management. 2018(10): 11-14.

[3] WANG Shui, CAI An-juan, QU Chang-sheng. Strengthen Soil Pollution Prevention and Control to Ensure the Safety of Soil Environment The Interpretation of the Work Plan of Soil Pollution Prevention and Control for Jiangsu Province [J]. Environmental monitoring and forewarning. 2017 (2): 1-5.

[4] QIU Lingling. Discussion on soil environmental management and remediation of contaminated sites [J]. Environment and Development. 2020, 32 (07): 228-229.

[5] YANG Haibing. Design and Research on decision system of soil heavy metal pollution control in the process of mine exploration and development [J]. Environmental Science and Management. 2021, 46 (03): 84-89. 